Research Article

\title{
Complex Dynamics of Credit Risk Contagion with Time-Delay and Correlated Noises
}

\author{
Tingqiang Chen, ${ }^{1}$ Xindan $\mathrm{Li}^{2}$ and Jianmin $\mathrm{He}^{3}$ \\ ${ }^{1}$ School of Economics and Management, Nanjing Tech. University, Nanjing 211816, China \\ ${ }^{2}$ School of Management and Engineering, Nanjing University, Nanjing 210093, China \\ ${ }^{3}$ School of Economics and Management, Southeast University, Nanjing 211189, China
}

Correspondence should be addressed to Tingqiang Chen; tingqiang88888888@163.com

Received 26 September 2013; Accepted 27 January 2014; Published 20 March 2014

Academic Editor: Leszek Gasinski

Copyright (C) 2014 Tingqiang Chen et al. This is an open access article distributed under the Creative Commons Attribution License, which permits unrestricted use, distribution, and reproduction in any medium, provided the original work is properly cited.

\begin{abstract}
The stochastic time-delayed system of credit risk contagion driven by correlated Gaussian white noises is investigated. Novikov's theorem, the time-delay approximation, the path-integral approach, and first-order perturbation theory are used to derive timedelayed Fokker-Planck model and the stationary probability distribution function of the dynamical system of credit risk contagion in the financial market. Using the method of numerical simulation, the Hopf bifurcation and chaotic behaviors of credit risk contagion are analyzed when time-delay and nonlinear resistance coefficient are varied and the effects of time-delay, nonlinear resistance and the intensity and the correlated degree of correlated Gaussian white noises on the stationary probability distribution of credit risk contagion are investigated. It is found that, as the infectious scale of credit risk and the wavy frequency of credit risk contagion are increased, the stability of the system of credit risk contagion is reduced, the dynamical system of credit risk contagion gives rise to chaotic phenomena, and the chaotic area increases gradually with the increase in time-delay. The nonlinear resistance only influences the infectious scale and range of credit risk, which is reduced when the nonlinear resistance coefficient increases. In addition, the curve of the stationary probability distribution is monotone decreasing with the increase in parameters value of time-delay, nonlinear resistance, and the intensity and the correlated degree of correlated Gaussian white noises.
\end{abstract}

\section{Introduction}

In the last few years, complex nonlinear dynamics analysis approach has provided an alternative scientific methodology to understand the highly complex nonlinear dynamics of the modern economic and financial systems [1-9]. Complex nonlinear dynamical phenomena have been investigated in the real financial and economic system, including irregular growth [10], exchange rate fluctuation [11], chaotic stock market behaviors [12], business cycle [13], bullwhip effect [14], and risk contagion $[8,9]$. The aforementioned phenomena present complex dynamical behavior, involving Hopf bifurcation, inverse bifurcation, chaos, and fractals. Among these behavior types, chaos and bifurcation are complex phenomena that exist in the nonlinear financial system and are important issues in economic and financial dynamics research [4]. In the field of finance, given the interaction among nonlinear factors, along with the increasing complexity of all kinds of financial problems and the evolution process from low dimensions to high dimensions, diversity and complexity have become explicit in the internal structure of the financial system, which consequently exhibits extremely complicated phenomena and external characteristics $[1,2]$, such as chaos phenomena and Hopf bifurcations of the economic and financial system $[3,5,8,9,15,16]$. For a credit risk contagion system, structural instability is inherent. Moreover, any appropriate disturbance can induce a sudden "qualitative" or topological change in the dynamic properties of such a system. Therefore, a study of complex nonlinear dynamics of credit risk contagion can describe the effect of the internal complexity of credit risk contagion system as well as providing theoretical and practical guidance to credit risk management.

Recently, stochastic systems with time-delay have attracted much attention in many fields, such as biological 
systems [17] and economic and financial phenomena $[8,9,18-20]$. It is well known that time-delay is an important factor of mathematical financial models; recent research on financial complex dynamics seems to be generating renewed interest in stochastic differential equations with time-delay terms. In fact, the financial dynamical system described by stochastic delay differential equations occupies a place of central importance in economic and financial area. This is because some economic and financial phenomena cannot be exhaustively described purely using differential equations. In the real financial market, time-delay usually arises because the speed of the transmission of information and noise and that of the transport of matter and energy are finite [21]. It is frequently encountered in real financial systems and very often is the main cause for instability of the financial system. The system of credit risk contagion is known to be a very complicated nonlinear system that is concerned with people and numerous complex factors, in which time-delay is also inherent [9]. In view of these, the issue of time-delay stochastic financial system is a topic of great theoretical and practical importance, and studies of credit risk contagion show that time-delays create a wide variety of dynamic behaviors including Hopf bifurcation and chaotic behaviors. Thus stochastic model of credit risk contagion with the time-delay needs to be investigated, and studying the effect of time-delay on credit risk contagion is necessary.

In addition, noise is prevalent in the financial system [22-27]. Noise makes financial markets possible, but it also makes such markets imperfect [22]. Moreover, the existence of noise is systematic [27]. Therefore, noise cannot be ignored in financial research. In the last few years, the effects of correlations between additive and multiplicative noise on stochastic systems have attracted the attention of many researchers [28-34]. In the real credit risk contagion, the existence of correlated noises and their interactive driving is also prevalent and very often is the main cause for stochastic default of credit activity participants. However, few works are devoted to the financial system driven by correlated noises. In view of this, the studying of credit risk contagion with driving of correlated noises is necessary. Studying the effects of correlations between additive and multiplicative noise on credit risk contagion has great theoretical and practical importance.

In this paper, the complex stochastic dynamics phenomena of credit risk contagion with time-delay and correlated noises are investigated. We extend the method presented in [35-38] to stochastic time-delayed model of credit risk contagion driven by correlated Gaussian white noises. The time-delayed Fokker-Planck model of credit risk contagion is obtained by using Novikov's theorem in Section 2. Then, in Section 3, the deterministic Hopf bifurcation and chaotic behaviors of credit risk contagion are investigated by numerical simulations. In Section 4, methods of the time-delay approximation, the path-integral approach, and first-order perturbation theory are applied to obtain the approximate stationary probability distribution of credit risk contagion in the financial market, and numerical simulations are performed to check the validity of analytic results. Finally, we conclude the paper in Section 5.

\section{The Fokker-Planck Model of Credit Risk Contagion in the Financial Market}

In this paper we will report a microscopic model of credit risk contagion in the financial market with time-delay and correlated noises, which aims at modelling the complex economic and social phenomena and investigating the complex nonlinear dynamics behavior in the process of credit risk contagion by considering the effect of time-delay and correlated noises.

The time-delay in the process of information gathering, recognizing, and transmission will be inevitable in the financial system. Particularly, in the process of remote transmission, the time-delay will be more evident $[8,9]$. Moreover, the synergistic effect of noise and time-delay is universal in the financial market, which facilitates complex nonlinear dynamics behavior $[8,9,39]$. We thus assume that the complex network connections among credit activity participants are Newman-Watts length scale connections and long-distance connections, which represent, respectively, direct business relation and other indirect relations. Earlier we investigated nonlinear stochastic system with timedelay properties in [8] but ignored the study of noise disturbance. Thus we will represent the model of credit risk contagion with time-delay and correlated noises as follows:

$$
\begin{aligned}
\frac{d N(t)}{d t}= & \lambda_{1} N(t)+\lambda_{2} N(t-\tau)-\mu \xi\left[\lambda_{2} N(t-\tau)\right]^{2} \\
& +N(t) \psi(t)+\eta(t),
\end{aligned}
$$

where $N(t)$ describes a state variable of credit risk contagion, which denotes the number of credit activity participants that are infected by credit risk in the financial market; $\lambda_{1}$ and $\lambda_{2}$ are the effective contagion rate of credit risk among different credit activity participants that correspond, respectively, to direct business relation and other indirect relations in the financial market; $\tau$ is the time-delay of credit risk contagion among different credit activity participants corresponding to other indirect relations in the financial market; $\xi$ refers to the Nelength scale; $\mu$ is the nonlinear resistance coefficient of the relationship network comprising credit activity participants in the financial market, which mainly reflect the heterogeneity among credit activity participants, including products and psychological behavior; $\psi(t)$ and $\eta(t)$ are noise terms. We assume $\lambda_{1}>\lambda_{2}$ in (1). In fact, $d N(t) / d t=$ $\lambda_{1} N(t)+\lambda_{2} N(t-\tau)-\mu \xi\left[\lambda_{2} N(t-\tau)\right]^{2}$ is the drift function of credit risk contagion and is also the deterministic part of (1). Obviously, (1) is a Stratonovich stochastic differential equation.

In the extant literature on finance, a number of studies adopt Gaussian white noise to analyze its effect on the economic and financial system, such as $[39,40]$. Therefore, in this paper, we also adopt Gaussian white noise to investigate its effect on credit risk contagion. We assume the noise terms 
$\psi(t)$ and $\eta(t)$ to be correlated Gaussian white noises with zero mean and

$$
\begin{gathered}
\left\langle\psi(t) \psi\left(t^{\prime}\right)\right\rangle=2 \sigma \delta\left(t-t^{\prime}\right), \\
\left\langle\eta(t) \eta\left(t^{\prime}\right)\right\rangle=2 \rho \delta\left(t-t^{\prime}\right), \\
\left\langle\psi(t) \eta\left(t^{\prime}\right)\right\rangle=\left\langle\eta(t) \psi\left(t^{\prime}\right)\right\rangle=2 \alpha \sqrt{\sigma \rho} \delta\left(t-t^{\prime}\right),
\end{gathered}
$$

where $\alpha$ is the correlated degree between the noise terms $\psi(t)$ and $\eta(t) . \sigma$ and $\rho$ are the strength of the noise terms $\psi(t)$ and $\eta(t)$, respectively. $\delta$ is an autocorrelation function. Therefore, the stochastic fluctuation of credit risk contagion can be depicted by $\alpha$ correlated Gaussian white noises $\psi(t)$ and $\eta(t), \delta$ autocorrelative Gaussian white noise $\psi(t)$, and $\delta$ autocorrelative Gaussian white noise $\eta(t)$.

We let $p(N, t)=\left\langle\delta\left(N(t)-N^{\prime}(t)\right)\right\rangle$ denote the probability density of credit risk contagion defined by (1). Therefore, a general delayed contagion effect of credit risk can describe the differentiating $p(N, t)$ as follows:

$$
\begin{aligned}
& \frac{\partial p(N, t)}{\partial t} \\
& =-\left\langle\frac{\partial}{\partial N} \delta\left(N(t)-N^{\prime}(t)\right) \frac{d N(t)}{d t}\right\rangle \\
& =-\frac{\partial}{\partial N}\left\langle\left[\lambda_{1} N(t)+\lambda_{2} N(t-\tau)-\mu \xi\left[\lambda_{2} N(t-\tau)\right]^{2}\right]\right. \\
& \left.\quad \times \delta\left(N(t)-N^{\prime}(t)\right)\right\rangle \\
& -\frac{\partial}{\partial N}\left\langle N(t) \psi(t) \delta\left(N(t)-N^{\prime}(t)\right)\right\rangle \\
& -\frac{\partial}{\partial N}\left\langle\eta(t) \delta\left(N(t)-N^{\prime}(t)\right)\right\rangle .
\end{aligned}
$$

We assume that $P\left(N, t ; N_{\tau}, t-\tau\right)$ is a joint probability density. For convenience, $t$ is dropped since it is the same for all variables $N(t)$ and $N^{\prime}(t)$, and $N_{\tau}$ denotes the time-delayed state variable $N(t-\tau)$. Thus, (3) can be rewritten as

$$
\begin{aligned}
& \frac{\partial p(N, t)}{\partial t} \\
& =\frac{\partial}{\partial N} \int_{0}^{\infty}\left[\lambda_{1} N+\lambda_{2} N_{\tau}-\mu \xi\left[\lambda_{2} N_{\tau}\right]^{2}\right] \\
& \quad \times P\left(N, t ; N_{\tau}, t-\tau\right) d N_{\tau} \\
& \quad-\frac{\partial}{\partial N} \int_{0}^{\infty} N\left\langle\psi(t) \delta\left(N-N^{\prime}\right) \delta\left(N_{\tau}-N_{\tau}^{\prime}\right)\right\rangle d N_{\tau} \\
& \quad-\frac{\partial}{\partial N} \int_{0}^{\infty}\left\langle\eta(t) \delta\left(N-N^{\prime}\right) \delta\left(N_{\tau}-N_{\tau}^{\prime}\right)\right\rangle d N_{\tau}
\end{aligned}
$$

According to Novikov's theorem [38, 41, 42], we can calculate $\left\langle\psi(t) \delta\left(N-N^{\prime}\right) \delta\left(N_{\tau}-N_{\tau}^{\prime}\right)\right\rangle$ and $\langle\eta(t) \delta(N-$ $\left.\left.N^{\prime}\right) \delta\left(N_{\tau}-N_{\tau}^{\prime}\right)\right\rangle$ as follows:

$$
\begin{aligned}
& \left\langle\psi(t) \delta\left(N-N^{\prime}\right) \delta\left(N_{\tau}-N_{\tau}^{\prime}\right)\right\rangle \\
& =2 \sigma \int_{0}^{t} \delta\left(t-t^{\prime}\right) \\
& \times\left\langle\frac{\partial\left(\delta\left(N-N^{\prime}\right)\right) \delta\left(N_{\tau}-N_{\tau}^{\prime}\right)}{\partial N} \frac{\partial N}{\partial \psi(t)}\right\rangle d t^{\prime} \\
& +2 \sigma \int_{0}^{t} \delta\left(t-t^{\prime}\right) \\
& \times\left\langle\frac{\partial\left(\delta\left(N-N^{\prime}\right)\right) \delta\left(N_{\tau}-N_{\tau}^{\prime}\right)}{\partial N_{\tau}} \frac{\partial N_{\tau}}{\partial \psi(t)}\right\rangle d t^{\prime} \\
& +2 \alpha \sqrt{\sigma \rho} \\
& \times \int_{0}^{t} \delta\left(t-t^{\prime}\right) \\
& \times\left\langle\frac{\partial\left(\delta\left(N-N^{\prime}\right)\right) \delta\left(N_{\tau}-N_{\tau}^{\prime}\right)}{\partial N} \frac{\partial N}{\partial \eta(t)}\right\rangle d t^{\prime} \\
& +2 \alpha \sqrt{\sigma \rho} \\
& \times \int_{0}^{t} \delta\left(t-t^{\prime}\right) \\
& \times\left\langle\frac{\partial\left(\delta\left(N-N^{\prime}\right)\right) \delta\left(N_{\tau}-N_{\tau}^{\prime}\right)}{\partial N_{\tau}} \frac{\partial N_{\tau}}{\partial \eta(t)}\right\rangle d t^{\prime} .
\end{aligned}
$$

According to literature [38], for all $\alpha>0$, we can get

$$
\begin{gathered}
\frac{\partial N_{\tau}}{\partial \psi(t)}=\frac{\partial N_{\tau}}{\partial \eta(t)}=0, \\
\frac{\partial N}{\partial \psi(t)}=\frac{1}{2} N, \\
\frac{\partial N}{\partial \eta(t)}=\frac{1}{2} .
\end{gathered}
$$

Thus, (5) can be rewritten as

$$
\begin{aligned}
\langle\psi(t) & \left.\delta\left(N-N^{\prime}\right) \delta\left(N_{\tau}-N_{\tau}^{\prime}\right)\right\rangle \\
= & -\sigma \frac{\partial}{\partial N} N\left\langle\delta\left(N-N^{\prime}\right) \delta\left(N_{\tau}-N_{\tau}^{\prime}\right)\right\rangle \\
& -\alpha \sqrt{\sigma \rho} \frac{\partial}{\partial N}\left\langle\delta\left(N-N^{\prime}\right) \delta\left(N_{\tau}-N_{\tau}^{\prime}\right)\right\rangle \\
= & -\sigma \frac{\partial}{\partial N} N p\left(N, t ; N_{\tau}, t-\tau\right) \\
& -\alpha \sqrt{\sigma \rho} \frac{\partial}{\partial N} p\left(N, t ; N_{\tau}, t-\tau\right) .
\end{aligned}
$$


Similarly, we can obtain

$$
\begin{aligned}
\langle\eta(t) & \left.\delta\left(N-N^{\prime}\right) \delta\left(N_{\tau}-N_{\tau}^{\prime}\right)\right\rangle \\
= & -\rho \frac{\partial}{\partial N} p\left(N, t ; N_{\tau}, t-\tau\right) \\
& -\alpha \sqrt{\sigma \rho} \frac{\partial}{\partial N} N p\left(N, t ; N_{\tau}, t-\tau\right) .
\end{aligned}
$$

We assume that $h\left(N, N_{\tau}\right)=\lambda_{1} N(t)+\lambda_{2} N(t-\tau)-$ $\mu \xi\left[\lambda_{2} N(t-\tau)\right]^{2}, g_{1}\left(N, N_{\tau}\right)=N(t)$, and $g_{2}\left(N, N_{\tau}\right)=$ 1. Substituting (7) and (8) into (4), we can get the timedelayed Fokker-Planck equation driven by correlated noises as follows:

$$
\begin{aligned}
\frac{\partial p(N, t)}{\partial t} & \int_{0}^{\infty}\left[-\frac{\partial}{\partial N} h\left(N, N_{\tau}\right)+\sigma \frac{\partial}{\partial N} g_{1}\left(N, N_{\tau}\right) \frac{\partial}{\partial N} g_{1}\left(N, N_{\tau}\right)\right. \\
& +\alpha \sqrt{\sigma \rho} \frac{\partial}{\partial N} g_{1}\left(N, N_{\tau}\right) \frac{\partial}{\partial N} g_{2}\left(N, N_{\tau}\right) \\
& +\rho \frac{\partial}{\partial N} g_{2}\left(N, N_{\tau}\right) \frac{\partial}{\partial N} g_{2}\left(N, N_{\tau}\right) \\
& \left.+\alpha \sqrt{\sigma \rho} \frac{\partial}{\partial N} g_{2}\left(N, N_{\tau}\right) \frac{\partial}{\partial N} g_{1}\left(N, N_{\tau}\right)\right] \\
& \times p\left(N_{\tau}, t-\tau \mid N, t\right) d N_{\tau} p(N, t),
\end{aligned}
$$

where $p\left(N_{\tau}, t-\tau \mid N, t\right)$ is the conditional probability density. Let

$$
\begin{aligned}
H\left(N, N_{\tau}\right)= & h\left(N, N_{\tau}\right)+\sigma g_{1}\left(N, N_{\tau}\right) \frac{\partial g_{1}\left(N, N_{\tau}\right)}{\partial N} \\
& +\alpha \sqrt{\sigma \rho} g_{2}\left(N, N_{\tau}\right) \frac{\partial g_{1}\left(N, N_{\tau}\right)}{\partial N} \\
& +\rho g_{2}\left(N, N_{\tau}\right) \frac{\partial g_{2}\left(N, N_{\tau}\right)}{\partial N} \\
& +\alpha \sqrt{\sigma \rho} g_{1}\left(N, N_{\tau}\right) \frac{\partial g_{2}\left(N, N_{\tau}\right)}{\partial N}, \\
G\left(N, N_{\tau}\right)= & \sigma g_{1}{ }^{2}\left(N, N_{\tau}\right)+2 \alpha \sqrt{\sigma \rho} g_{1}\left(N, N_{\tau}\right) g_{2}\left(N, N_{\tau}\right) \\
& +\rho g_{2}{ }^{2}\left(N, N_{\tau}\right) .
\end{aligned}
$$

Namely,

$$
\begin{gathered}
H\left(N, N_{\tau}\right)=\lambda_{1} N(t)+\lambda_{2} N(t-\tau)-\mu \xi\left[\lambda_{2} N(t-\tau)\right]^{2}+\sigma N \\
G\left(N, N_{\tau}\right)=\sigma N^{2}+2 \alpha \sqrt{\sigma \rho} N+\rho .
\end{gathered}
$$

So, the time-delayed Fokker-Planck equation driven by correlated noises can be rewritten as

$$
\begin{aligned}
\frac{\partial p(N, t)}{\partial t}=\int_{0}^{\infty} & {\left[-\frac{\partial}{\partial N} H\left(N, N_{\tau}\right)+\frac{\partial^{2}}{\partial N} G\left(N, N_{\tau}\right)\right] } \\
& \times p\left(N_{\tau}, t-\tau N, t\right) d N_{\tau} p(N, t) .
\end{aligned}
$$

\section{Hopf Bifurcation and Chaotic Behavior of Credit Risk Contagion in the Financial Market}

In (1) its deterministic part can be written as

$$
\frac{d N(t)}{d t}=\lambda_{1} N(t)+\lambda_{2} N(t-\tau)-\mu \xi\left[\lambda_{2} N(t-\tau)\right]^{2} .
$$

Equation (13) is also the drift function of credit risk contagion in the financial market, which is a time-delayed stochastic differential system. When the time-delay $\tau>$ 0 , (13) can be numerically calculated. We can describe the dynamics behaviors of evolution of credit risk contagion without noise effects and analyze their influencing factors by numerical calculating of (13). Let $\lambda_{1}=0.12, \lambda_{2}=0.07$, $\xi=3$, and the initial value $N_{0}=1$. Thus we can analyze the effects of parameters $\tau$ and $\mu$ to the dynamics behaviors of evolution of credit risk contagion without noise effects. Firstly, we analyze the effects of parameters $\tau$ to the dynamics behaviors of evolution of credit risk contagion when $\tau=0.04$. The time process diagrams and the deterministic bifurcation diagram of credit risk contagion are plotted in Figures 1 and 2 when $\tau$ is varied. Figure 1 depicts the time process of credit risk contagion under the influence of time-delay $\tau$. In Figure 1, with the increase in time-delay $\tau$, the infectious scale and range of credit risk are increased gradually, and the wavy frequency of credit risk contagion is also increased acutely, which shows that, with the increase in time-delay $\tau$, the stability of the system of credit risk contagion is reduced. In Figure 2, the deterministic bifurcation diagram of credit risk contagion is plotted as a function of $\tau$, which depicts chaotic behaviors of the system of credit risk contagion intuitively. It is seen that the infectious scale of credit risk in the equilibrium position of $N=323$ when $\tau<4.055$. For $4.055<\tau<4.8506$, the infectious scale and range of credit risk begin to jump and fluctuate. When $\tau>4.8506$, the dynamical system of credit risk contagion gives rise to Hopf bifurcation, inverse bifurcation, and chaos phenomena with an increase in time-delay $\tau$. Moreover, the chaotic area increases gradually with an increase in time-delay $\tau$. Figure 2 shows that, with the increasing of time-delay $\tau$, the predictability of the influencing degree of credit risk contagion reduced acutely.

In the real financial market, the effects of the nonlinear resistance coefficient on the infectious scale and range of credit risk have a decisive influence. Figure 3 depicts the influence of the evolution of credit risk contagion when $\tau=4$. In Figure 3, with the increase in nonlinear resistance coefficient $\mu$, the infectious scale and range of credit risk are reduced gradually, which is consistent with the result of 


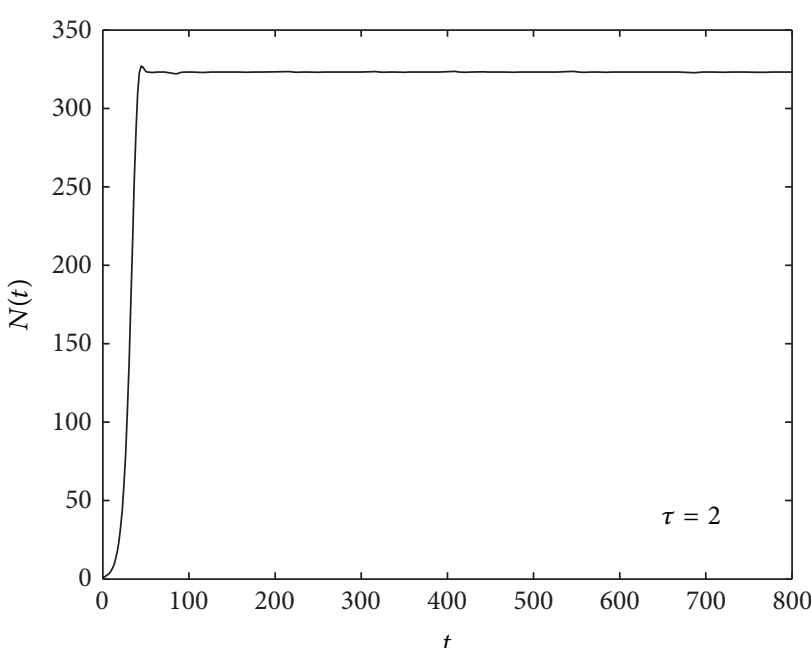

(a)

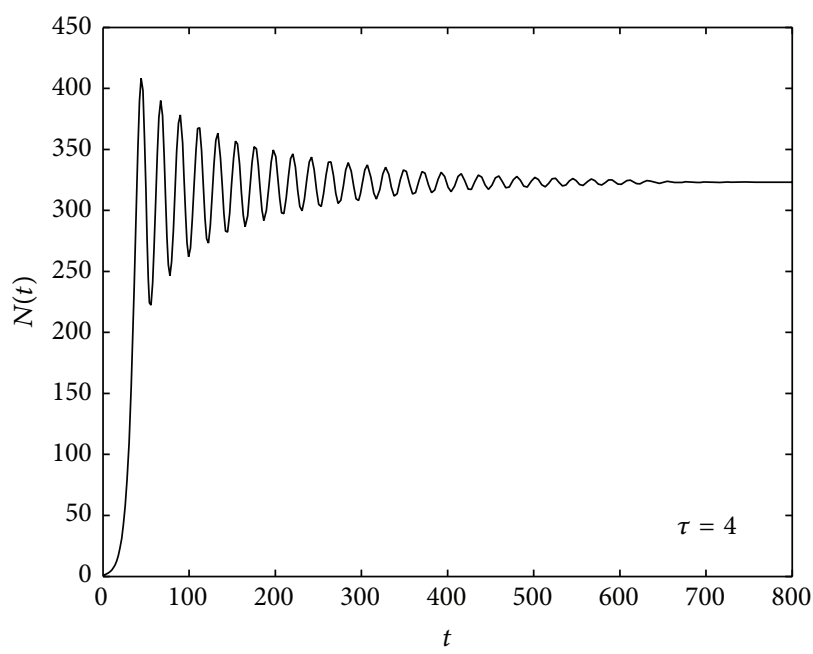

(c)

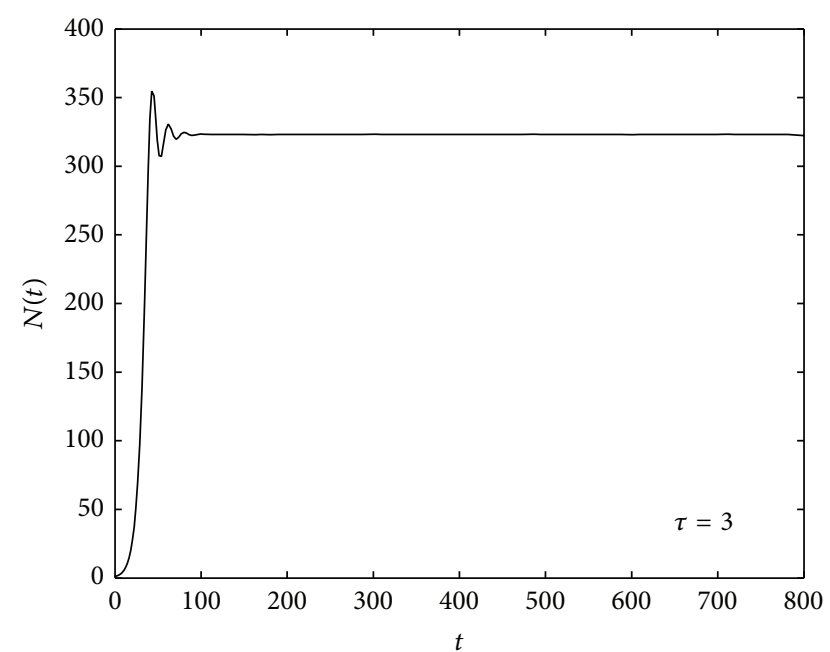

(b)

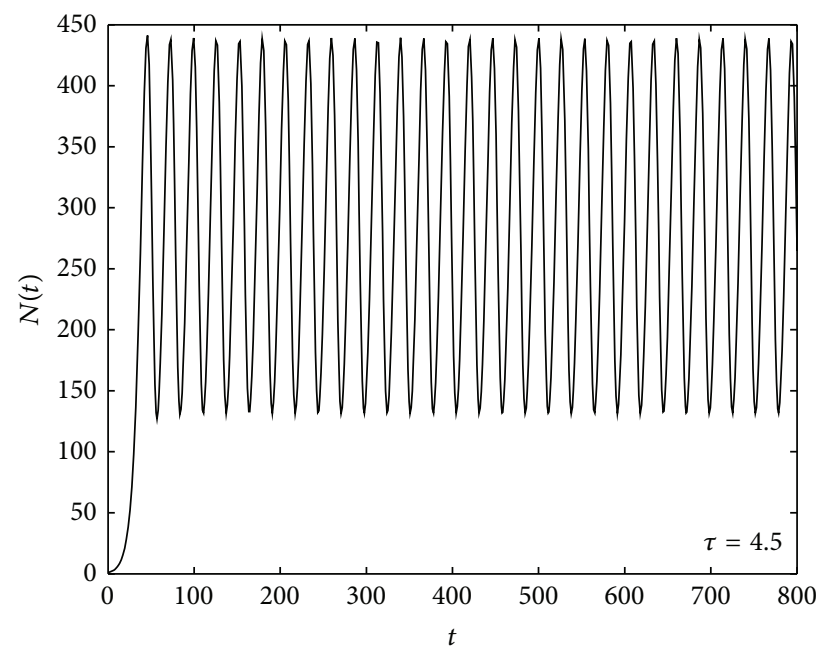

(d)

FIGURE 1: Time process diagrams of credit risk contagion in the financial market under the influence of time-delay $\tau$.

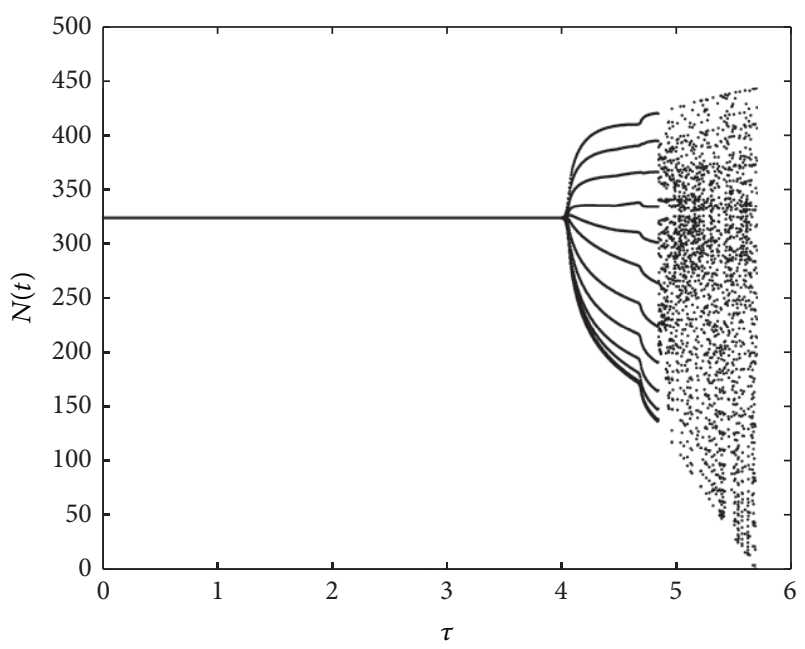

Figure 2: Bifurcation and chaotic properties of the dynamical system of credit risk contagion in the financial market when the time-delay $\tau$ is varied.
[9]. However, the wavy frequency of credit risk contagion is constant. Figure 4 depicts the effects of the nonlinear resistance coefficient $\mu$ on Hopf bifurcation and chaotic behaviors of the dynamical system of credit risk contagion. In Figure 4, with the increase in nonlinear resistance coefficient $\mu$, the equilibrium positions of the infectious scale and range of credit risk and the chaotic area are reduced gradually, but the point in time is fixed that the infectious scale and range of credit risk begin to jump and fluctuate, and the dynamical system of credit risk contagion gives rise to Hopf bifurcation, inverse bifurcation, and chaos phenomena.

\section{Stationary Probability Distribution of Credit Risk Contagion in the Financial Market}

In (12) we obtained the time-delayed Fokker-Planck equation driven by correlated noises to credit risk contagion in the financial market. This is a stochastic perturbed system with 


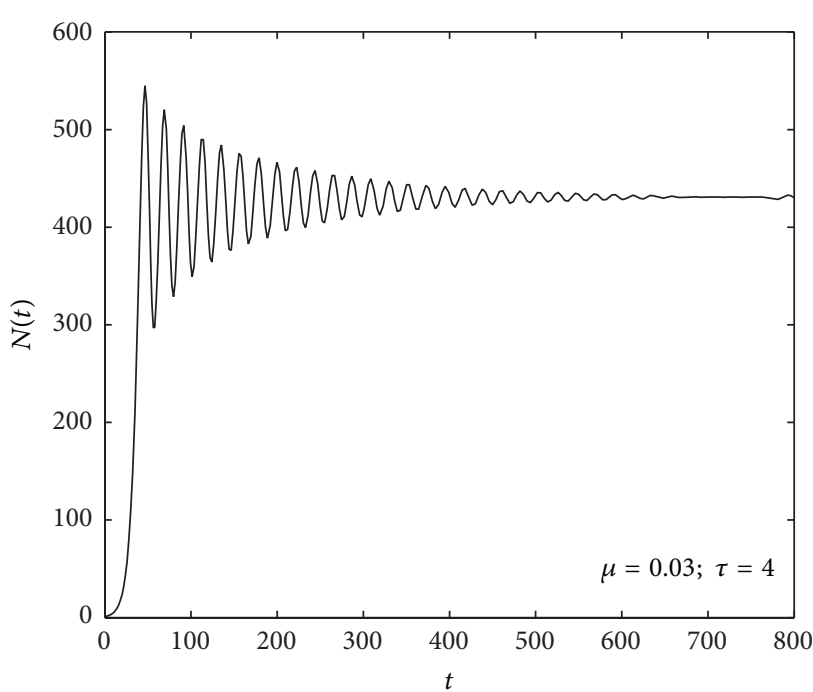

(a)

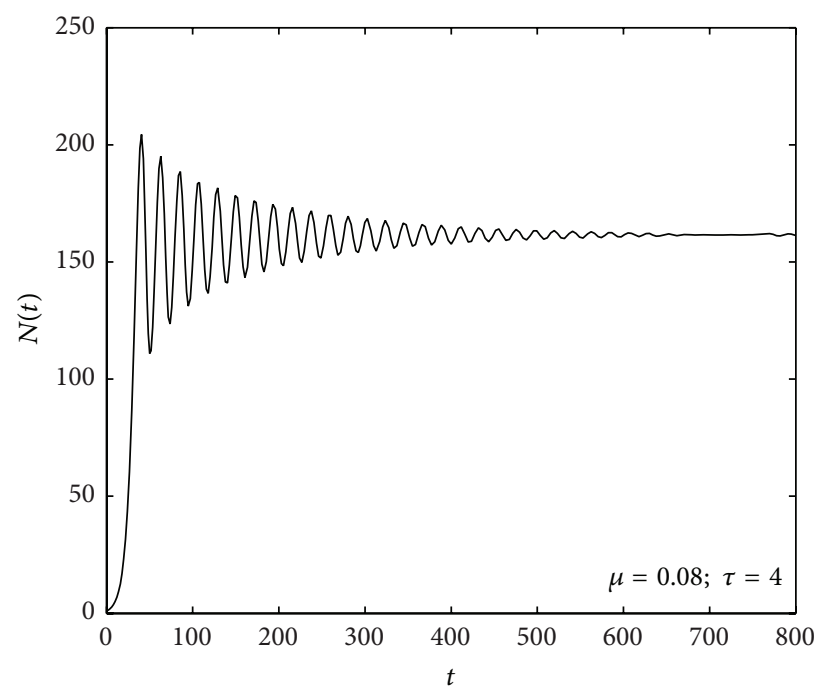

(c)

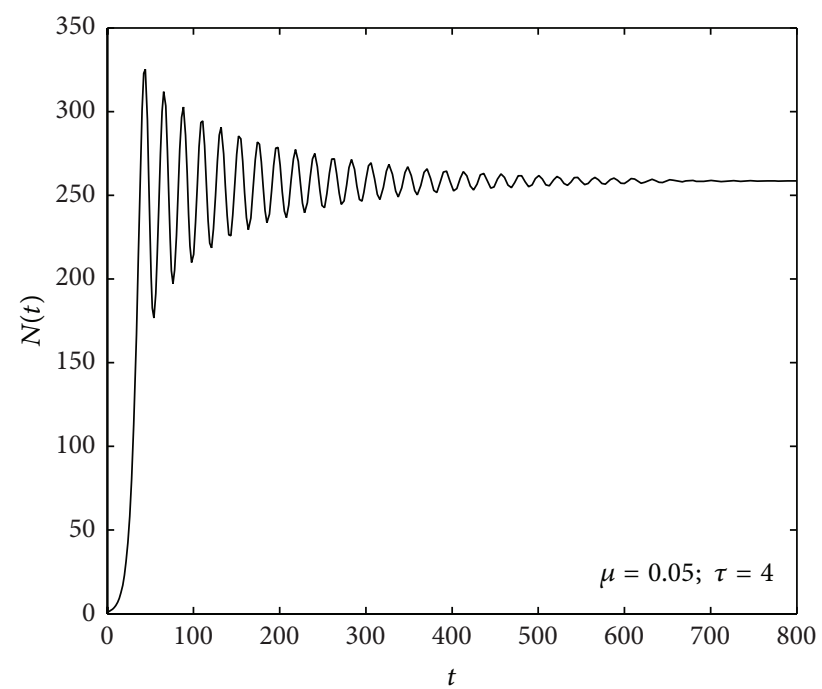

(b)

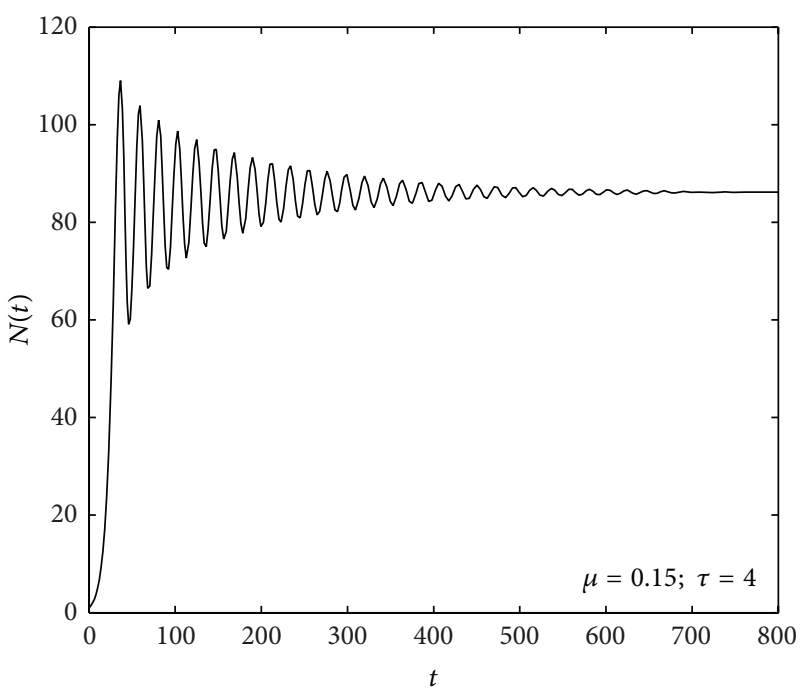

(d)

FIGURE 3: Time process diagrams of credit risk contagion in the financial market under the influence of the nonlinear resistance coefficient $\mu$.

time-delay. According to literatures $[35,36,38]$, the FokkerPlanck equation of the unperturbed system corresponding to (1) and (12) can be approximately written as

$$
\begin{aligned}
\frac{\partial p(N, t)}{\partial t}= & -\frac{\partial}{\partial N}[H(N, N) p(N, t)] \\
& +\frac{\partial^{2}}{\partial N}[G(N, N) p(N, t)],
\end{aligned}
$$

where the drift and diffusion coefficients of credit risk contagion in the financial market are

$$
\begin{gathered}
H(N, N)=\lambda_{1} N(t)+\lambda_{2} N(t)-\mu \xi\left[\lambda_{2} N(t)\right]^{2}+\sigma N, \\
G(N, N)=\sigma N^{2}+2 \alpha \sqrt{\sigma \rho} N+\rho .
\end{gathered}
$$

We assume that the stationary solution is $P_{\mathrm{st}}(N, t)$. Thus, the stationary solution $P_{\mathrm{st}}(N, t)$ of (1) can be analytically expressed as

$$
P_{\mathrm{st}}(N, t)=\frac{c}{A_{a}(N)} e^{\int_{0}^{N}\left(B_{a}\left(N^{\prime}\right) / A_{a}^{2}\left(N^{\prime}\right)\right) d N^{\prime}},
$$

where $A_{a}(N)=\left(\sqrt{\sigma N^{2}+2 \alpha \sqrt{\sigma \rho} N+\rho} /\left(1+\mu \xi \lambda_{2}^{2} N\right)\right)[1+$ $\left.\tau\left(2 \mu \xi \lambda_{2}^{2} N-\lambda_{2}\right) /\left(1+\mu \xi \lambda_{2}^{2} N\right)\right], B_{a}(N)=\left(\left(\lambda_{1} N+\right.\right.$ $\left.\left.\lambda_{2} N-\mu \xi \lambda_{2}{ }^{2} N^{2}\right) /\left(1+\mu \xi \lambda_{2}{ }^{2} N\right)\right)\left[1+\tau\left(2 \mu \xi \lambda_{2}{ }^{2} N-\lambda_{2}\right) /(1+\right.$ $\left.\mu \xi \lambda_{2}{ }^{2} N\right)$ ], and $c$ is the normalization constant. Thus, the 


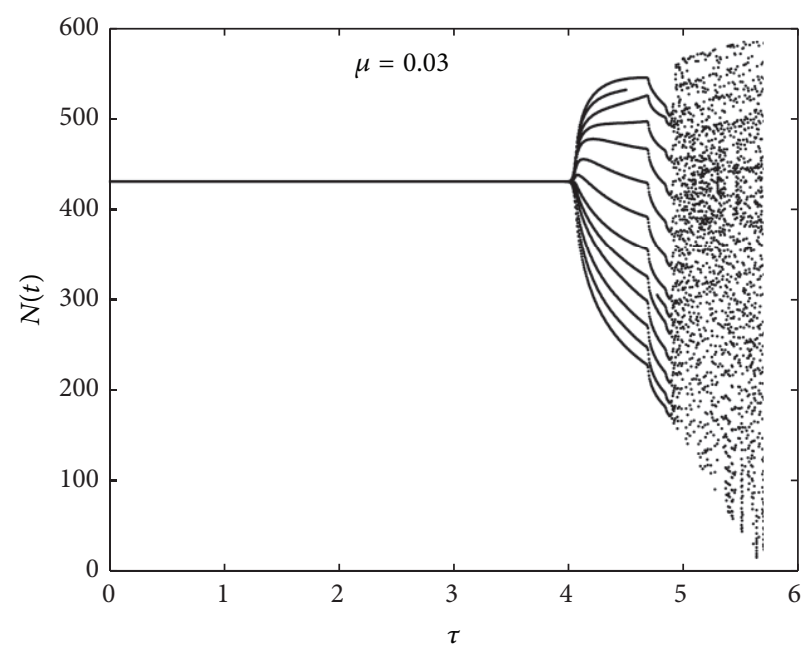

(a)

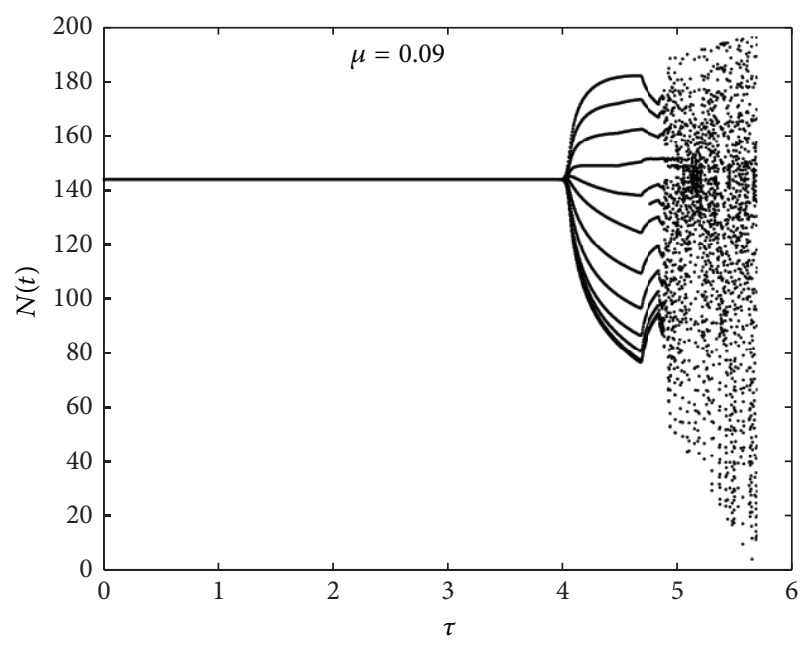

(c)

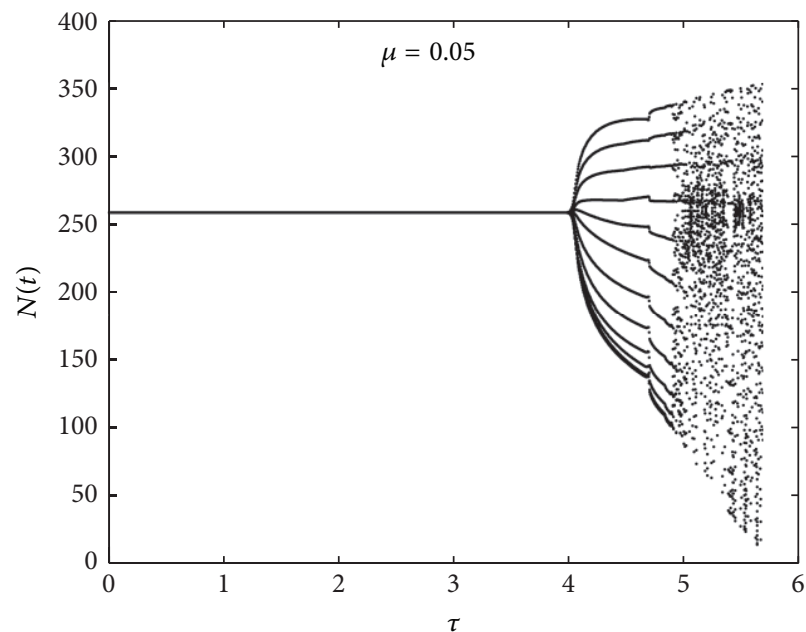

(b)

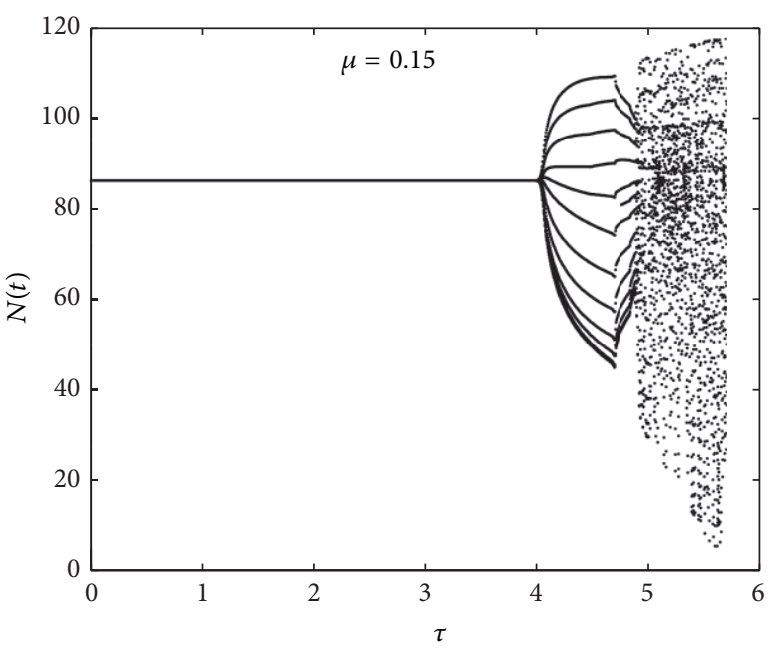

(d)

FIGURE 4: Bifurcation and chaotic properties of the dynamical system of credit risk contagion in the financial market when the time-delay $\tau$ is varied under the influence of the nonlinear resistance coefficient $\mu$.

stationary probability distribution of credit risk contagion corresponding to (1) can be calculated from (14) as follows:

$$
\begin{aligned}
P_{\text {st }}(N, t)= & \frac{c}{\left(\sqrt{\sigma N^{2}+2 \alpha \sqrt{\sigma \rho} N+\rho /}\left(1+\mu \xi \lambda_{2}{ }^{2} N\right)\right)\left[1+\tau\left(2 \mu \xi \lambda_{2}{ }^{2} N-\lambda_{2}\right) /\left(1+\mu \xi \lambda_{2}{ }^{2} N\right)\right]} \\
& \times e^{\left(\lambda_{1} N+\lambda_{2} N-\mu \xi \lambda_{2}{ }^{2} N^{2}\right)\left(1+\mu \xi \lambda_{2}{ }^{2} N\right) /\left(\sigma N^{2}+2 \alpha \sqrt{\sigma \rho} N+\rho\right)\left[1+\tau\left(2 \mu \xi \lambda_{2}{ }^{2} N-\lambda_{2}\right) /\left(1+\mu \xi \lambda_{2}{ }^{2} N\right)\right]} .
\end{aligned}
$$

According to (17), we can analyze the effects of parameters $\tau, \mu, \sigma, \rho$, and $\alpha$ on the stationary probability distribution of credit risk contagion through the numerical simulation. Thus the approximate analytical result of the stationary probability distribution $P_{\mathrm{st}}(N)$ as a function of the infectious scale and range of credit risk $N$ is plotted in Figures 5 and 6 when parameters $\tau, \mu, \sigma, \rho$, and $\alpha$ are varied. In Figure 5, with the increase in the infectious scale and range of credit risk
$N$, the stationary probability $P_{\text {st }}(N)$ is reduced gradually when parameters $\tau$ and $\mu$ are unvaried. From Figure 5(a), we find that, with the increase in time-delay $\tau$, the stationary probability $P_{\mathrm{st}}(N)$ is increased gradually when $N<200$. But when $N=200$, with the increase in time-delay $\tau$, the stationary probability $P_{\text {st }}(N)$ is unaltered. When $N=200$, with the increase in time-delay $\tau$, the stationary probability $P_{\mathrm{st}}(N)$ is reduced gradually. Thus Figure 5(a) shows that 


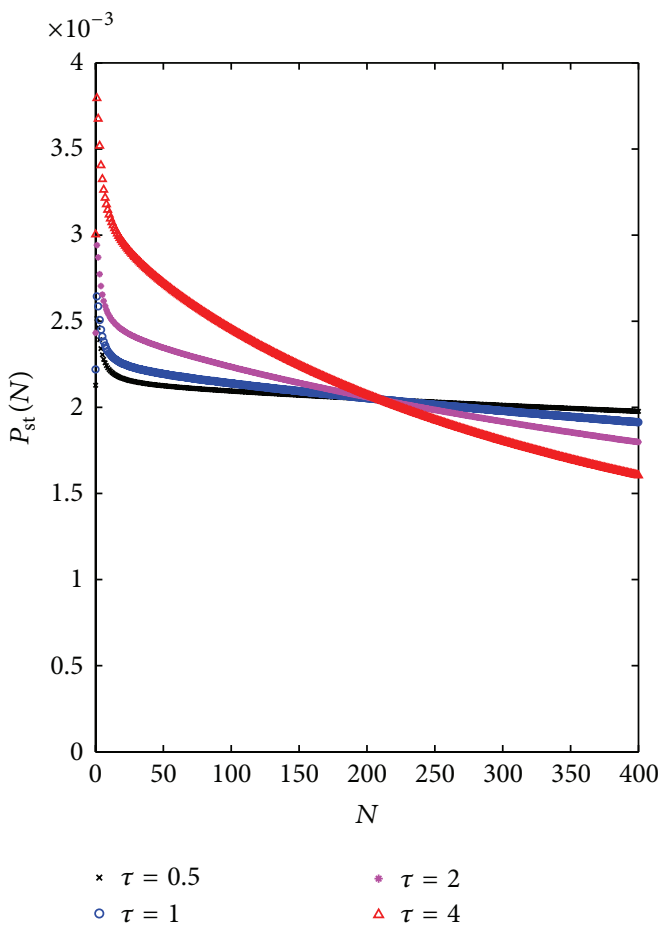

(a)

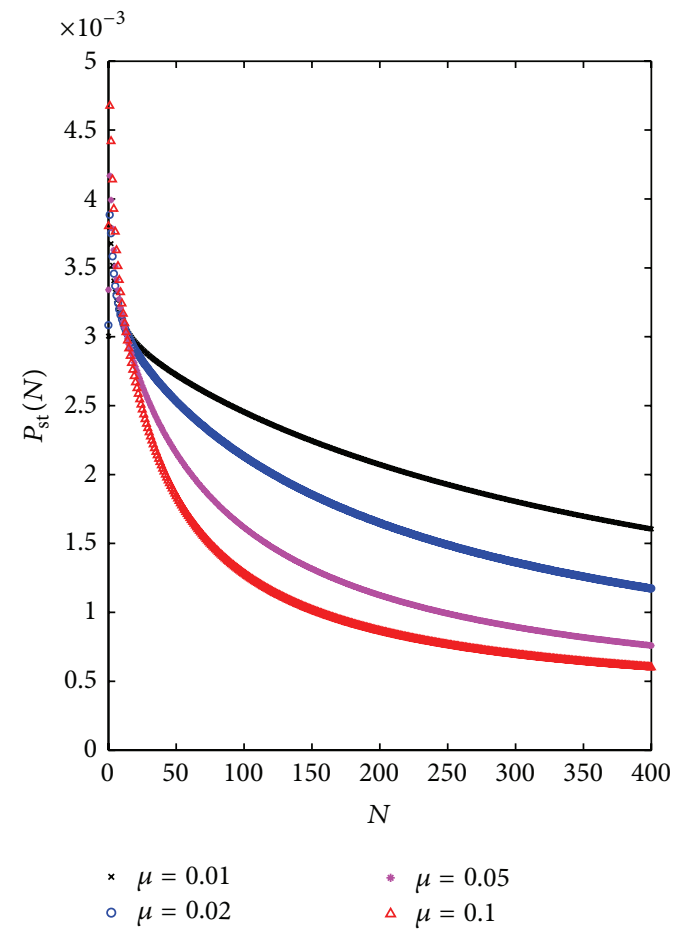

(b)

FIGURE 5: The stationary probability distribution function of credit risk contagion $P_{\text {st }}(N)$ is plotted as a function of $N$ when $\tau$ and $\mu$ are varied. The parameters are chosen as $\sigma=0.5, \rho=0.6, \alpha=0.3, \xi=3, \lambda_{1}=0.15$, and $\lambda_{2}=0.08$. (a) $\mu=0.01$. (b) $\tau=4$.

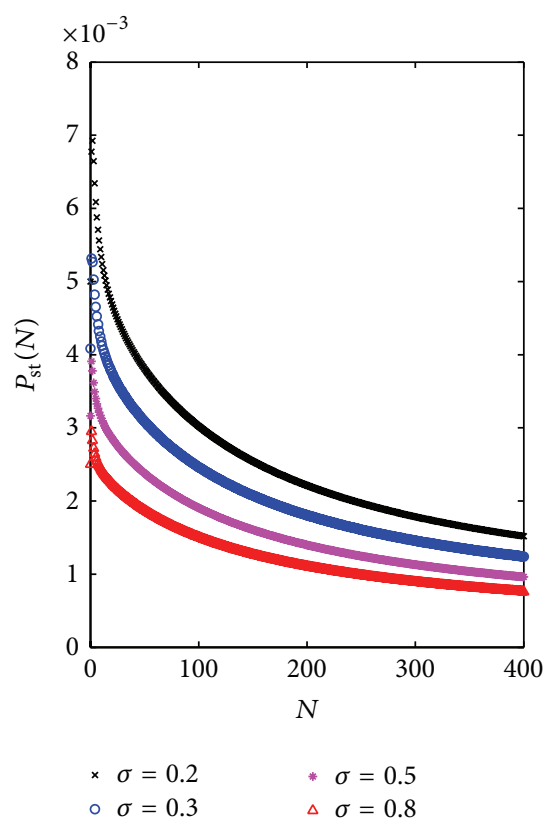

(a)

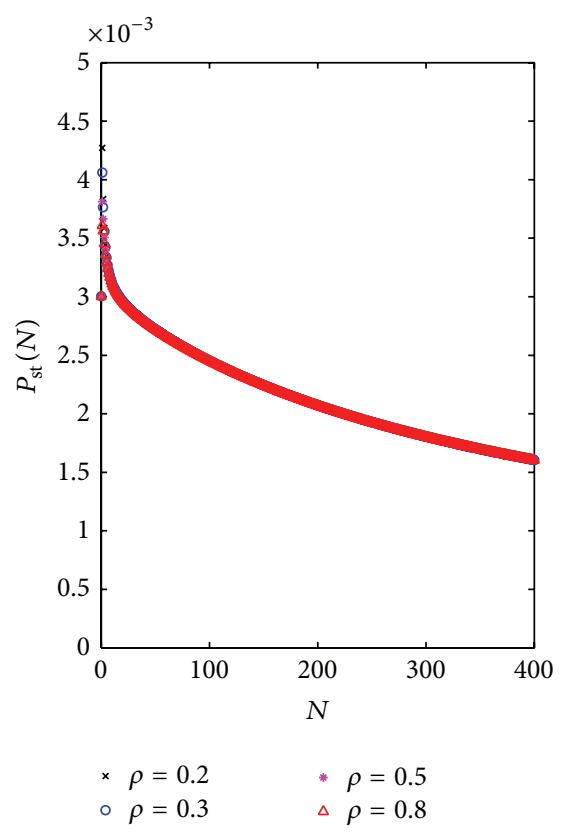

(b)

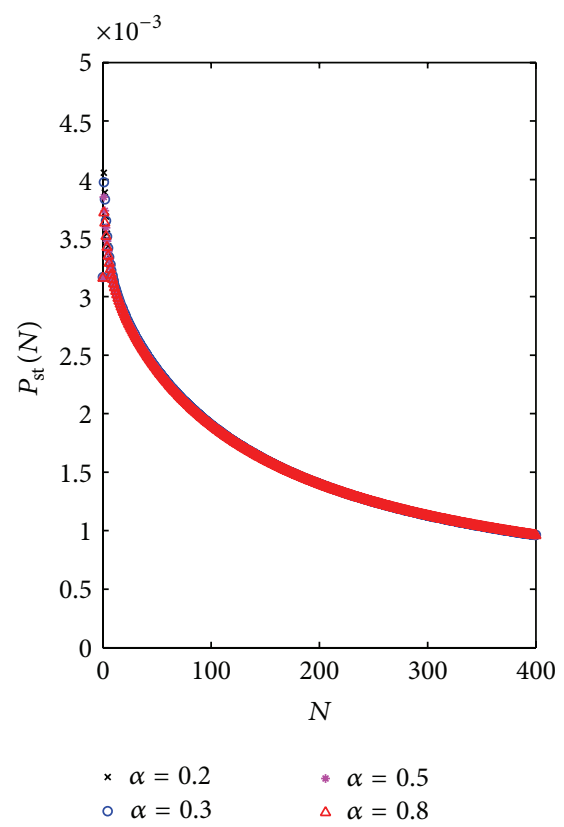

(c)

Figure 6: The stationary probability distribution function of credit risk contagion $P_{\text {st }}(N)$ is plotted as a function of $N$ when $\sigma, \rho$, and $\alpha$ are varied. The parameters are chosen as $\tau=4, \mu=0.03, \xi=3, \lambda_{1}=0.15$, and $\lambda_{2}=0.08$. (a) $\rho=0.6, \alpha=0.4$. (b) $\sigma=0.5$ and $\alpha=0.4$. (c) $\sigma=0.5$, and $\rho=0.6$. 


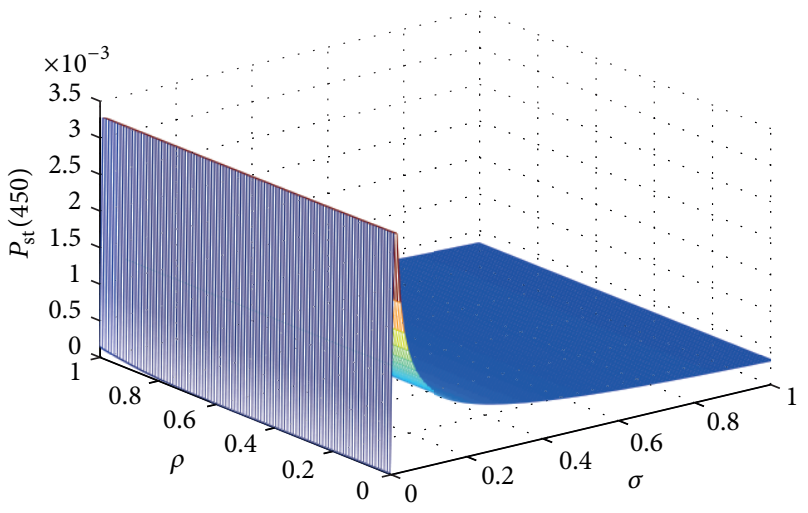

FIGURE 7: The three-dimensional diagram of the stationary probability distribution function of credit risk contagion $P_{\mathrm{st}}(N)$ as a function of $\sigma$ and $\rho$ when $N=450, \tau=4, \mu=0.03, \xi=3, \lambda_{1}=0.15$, $\lambda_{2}=0.08$, and $\alpha=0.4$.

the effects of time-delay $\tau$ on the stationary probability $P_{\mathrm{st}}(N)$ reveal a critical point $N^{*}$. Moreover, time-delay will not influence the stationary probability $P_{\mathrm{st}}(N)$ when the infectious scale and range of credit risk $N$ reach the threshold value $N^{*}$. In Figure 5(b), with the increase in the nonlinear resistance coefficient $\mu$, the stationary probability $P_{\text {st }}(N)$ is increased gradually when $N<15$. But when $N>15$, with the increase in the nonlinear resistance coefficient $\mu$, the stationary probability $P_{\mathrm{st}}(N)$ is reduced gradually. Thus the effects of the nonlinear resistance coefficient $\mu$ on the stationary probability $P_{\text {st }}(N)$ also reveal a critical point $N^{* \prime}$.

Figure 6 depicts the effects of the noise intensities, $\sigma$ and $\rho$ and the correlated degree $\alpha$ between Gaussian white noises $\psi(t)$ and $\eta(t)$ on the stationary probability of credit risk contagion $P_{\mathrm{st}}(N)$. In Figure 6(a), with the increase in the intensity $\sigma$ of the noise term $\psi(t)$, the stationary probability $P_{\mathrm{st}}(N)$ is reduced gradually. In Figures 6(b) and 6(c), it is clear that the effects of the intensity $\rho$ of the noise term $\eta(t)$ and the correlated degree $\alpha$ between noises $\psi(t)$ and $\eta(t)$ on the stationary probability $P_{\mathrm{st}}(N)$ are very slight. The threedimensional diagram of $P_{\mathrm{st}}(N)$ as a function of the intensities $\sigma$ and $\rho$ of Gaussian noises $\psi(t)$ and $\eta(t)$ is shown in Figure 7 when the infectious scale and range of credit risk $N=450$. From Figure 7, it is seen that the cure of the stationary probability $P_{\mathrm{st}}(450)$ is changed slightly with the increase in the intensity $\rho$ of Gaussian white noise $\eta(t)$ but is significantly changed with the increase in the intensity $\sigma$ of Gaussian white noise $\psi(t)$.

\section{Conclusion}

In this paper the complex stochastic dynamics phenomena of credit risk contagion with time-delay driven by correlated Gaussian white noises are investigated. The time-delayed Fokker-Planck model of credit risk contagion driven by correlated Gaussian white noises is obtained by using Novikov's theorem. Moreover, the stationary probability distribution function of the dynamical system of credit risk contagion is derived by combining the methods of the time-delay approximation, the path-integral approach, and first-order perturbation theory. Using the method of numerical simulation, the Hopf bifurcation and chaotic behaviors of credit risk contagion are analyzed when time-delay and nonlinear resistance coefficient are varied. The numerical simulations show that, with the increase in time-delay, the infectious scale and range of credit risk are increased gradually and the wavy frequency of credit risk contagion is increased acutely. Moreover, the stability of the system of credit risk contagion is reduced, the dynamical system of credit risk contagion gives rise to chaotic phenomena, and the chaotic area increases gradually with the increase in time-delay. In addition, the effects of time-delay, nonlinear resistance, and the intensity and the correlated degree of correlated Gaussian white noises on the stationary probability distribution of credit risk contagion are investigated by the numerical simulations. It is found that the curve of the stationary probability distribution is monotone decreasing with the increase in parameters value of time-delay, nonlinear resistance, and the intensity and the correlated degree of correlated Gaussian white noises.

However, further studies could expand the present work to cover other topics. For example, in the real world, types of noises usually affect the process of credit risk contagion and its dynamics behavior, such as Poissonian white noise, combined Gaussian and Poissonian noise, and colored noise. Therefore, our future study will focus on these issues.

\section{Conflict of Interests}

The authors declare that there is no conflict of interests regarding the publication of this paper.

\section{Acknowledgments}

The authors wish to express their gratitude to the referees for their invaluable comments. This work was supported by the National Natural Science Foundation of China Grant (nos. 70932003, 71201023, 71371051, and 71301078), the Humanities and Social Science Youth Foundation of the Ministry of Education of China (no.12YJC630101), the Funding of Jiangsu Innovation Program for Graduate Education (no. CXZZ120131).

\section{References}

[1] J. H. Ma and Y. S. Chen, "Study for the bifurcation topological structure and the global complicated character of a kind of nonlinear finance system-I," Applied Mathematics and Mechanics, vol. 22, no. 11, pp. 1240-1251, 2001.

[2] J. H. Ma and Y. S. Chen, "Study for the bifurcation topological structure and the global complicated character of a kind of nonlinear finance system-II," Applied Mathematics and Mechanics, vol. 22, no. 12, pp. 1375-1382, 2001.

[3] A. C.-L. Chian, E. L. Rempel, and C. Rogers, "Complex economic dynamics: chaotic saddle, crisis and intermittency," Chaos, Solitons and Fractals, vol. 29, no. 5, pp. 1194-1218, 2006.

[4] B. Xin, J. Ma, and Q. Gao, “The complexity of an investment competition dynamical model with imperfect information in a 
security market," Chaos, Solitons \& Fractals, vol. 42, no. 4, pp. 2425-2438, 2009.

[5] Q. Gao and J. Ma, "Chaos and Hopf bifurcation of a finance system," Nonlinear Dynamics, vol. 58, no. 1-2, pp. 209-216, 2009.

[6] W. Wu, Z. Chen, and Z. Yuan, "The evolution of a novel fourdimensional autonomous system: among 3-torus, limit cycle, 2torus, chaos and hyperchaos," Chaos, Solitons and Fractals, vol. 39, no. 5, pp. 2340-2356, 2009.

[7] W. Wu and Z. Chen, "Hopf bifurcation and intermittent transition to hyperchaos in a novel strong four-dimensional hyperchaotic system," Nonlinear Dynamics, vol. 60, no. 4, pp. 615-630, 2010.

[8] T. Chen, J. He, and Q. Yin, "Dynamics evolution of credit risk contagion in the CRT market," Discrete Dynamics in Nature and Society, vol. 2013, Article ID 206201, 9 pages, 2013.

[9] T. Chen, J. He, and J. Wang, "Bifurcation and chaotic behavior of credit risk contagion based on Fitzhugh-Nagumo system," International Journal of Bifurcation and Chaos in Applied Sciences and Engineering, vol. 23, no. 7, Article ID 1350117, 2013.

[10] R. H. Day, "Irregular growth cycles," American Economic Review, vol. 72, pp. 406-414, 1982.

[11] D. A. Hesieh, "Testing for nonlinear dependence in daily foreign exchange rates," Journal Business, vol. 62, pp. 339-359, 1989.

[12] D. A. Hesieh, "Chaos and nonlinear dynamics: application to financial markets,” The Journal of Finance, vol. 46, pp. 1839-1877, 1991.

[13] T. Puu and I. Sushko, "A business cycle model with cubic nonlinearity," Chaos, Solitons and Fractals, vol. 19, no. 3, pp. 597612, 2004.

[14] B. G. Xin, T. Chen, and J. H. Ma, "Neimark-Sacker bifurcation in a discrete-time financial system," Discrete Dynamics in Nature and Society, vol. 2010, Article ID 405639, 12 pages, 2010.

[15] S. Dowrick, R. Pitchford, and S. J. Turnovsky, Economic Growth and Macroeconomic Dynamics: Recent Developments in Economic Theory, Cambridge University Press, Cambridge, UK, 2004.

[16] R. Zhang, "Bifurcation analysis for a kind of nonlinear finance system with delayed feedback and its application to control of chaos," Journal of Applied Mathematics, vol. 2012, Article ID 316390, 18 pages, 2012.

[17] S. Gakkhar and A. Singh, "Complex dynamics in a prey predator system with multiple delays," Communications in Nonlinear Science and Numerical Simulation, vol. 17, no. 2, pp. 914-929, 2012.

[18] W.-C. Chen, "Dynamics and control of a financial system with time-delayed feedbacks," Chaos, Solitons and Fractals, vol. 37, no. 4, pp. 1198-1207, 2008.

[19] W.-C. Chen, "Nonlinear dynamics and chaos in a fractionalorder financial system," Chaos, Solitons and Fractals, vol. 36, no. 5, pp. 1305-1314, 2008.

[20] Y. Wang, Y. H. Zhai, and J. Wang, "Chaos and hopf bifurcation of a finance system with distributed time delay," International Journal of Applied Mathematics and Mechanics, vol. 6, pp. 1-13, 2010.

[21] D. Wu and S. Zhu, "Stochastic resonance in FitzHugh-Nagumo system wtih time-delayed feedback," Physics Letters A, vol. 372, no. 32, pp. 5299-5304, 2008.

[22] F. Black, "Noise," Journal of Finance, vol. 41, pp. 529-543, 1986.

[23] T. Odean, "Do investors trade too much?" American Economic Review, vol. 89, no. 5, pp. 1279-1298, 1999.
[24] B. M. Barber and T. Odean, "Trading is hazardous to your wealth: the common stock investment performance of individual investors," Journal of Finance, vol. 55, no. 2, pp. 773-806, 2000.

[25] A. Shleifer, Inefficient Markets: An Introduction to Behavioral Finance, Oxford University Press, New York, NY, USA, 2000.

[26] R. Tumarkin and R. F. Whitelaw, "News or noise? Internet postings and stock prices," Financial Analysts Journal, vol. 57, no. 3, pp. 41-51, 2001.

[27] B. Barber, T. Odean, and N. Zhu, "Do retail trades move markets?” Review of Financial Studies, vol. 22, pp. 151-186, 2009.

[28] G. Y. Liang, L. Cao, and D. J. Wu, "Approximate Fokker-Planck equation of system driven by multiplicative colored noises with colored cross-correlation," Physica A, vol. 335, no. 3-4, pp. 371384, 2004.

[29] Y. F. Jin and W. Xu, "Mean first-passage time of a bistable kinetic model driven by two different kinds of coloured noises," Chaos, Solitons and Fractals, vol. 23, no. 1, pp. 275-280, 2005.

[30] Y. F. Jin, W. Xu, and M. Xu, "Stochastic resonance in an asymmetric bistable system driven by correlated multiplicative and additive noise," Chaos, Solitons and Fractals, vol. 26, no. 4, pp. 1183-1187, 2005.

[31] D. Wu, X. Q. Luo, and S. Q. Zhu, "Stochastic system with coupling between non-Gaussian and Gaussian noise terms," Physica A, vol. 373, pp. 203-214, 2007.

[32] H. Q. Zhang, W. Xu, and Y. Xu, "The study on a stochastic system with non-Gaussian noise and Gaussian colored noise," Physica A, vol. 388, no. 6, pp. 781-788, 2009.

[33] R.-H. Shao and Y. Chen, "Stochastic resonance in time-delayed bistable systems driven by weak periodic signal," Physica A, vol. 388, no. 6, pp. 977-983, 2009.

[34] Z.-L. Jia, "Effects of time delay on transient behavior of a timedelayed metastable system subjected to cross-correlated noises," Physica A, vol. 387, no. 25, pp. 6247-6251, 2008.

[35] T. D. Frank, "Delay Fokker-Planck equations, perturbation theory, and data analysis for nonlinear stochastic systems with time delays," Physical Review E, vol. 71, no. 3, Article ID 031106, 2005.

[36] T. D. Frank, “Delay Fokker-Planck equations, Novikov's theorem, and Boltzmann distributions as small delay approximations," Physical Review E, vol. 72, no. 1, Article ID 011112, 2005.

[37] D. Wu and S. Zhu, "Stochastic resonance in a bistable system with time-delayed feedback and non-Gaussian noise," Physics Letters A, vol. 363, no. 3, pp. 202-212, 2007.

[38] H. Zhang, W. Xu, Y. Xu, and D. Li, "Stochastic time-delayed systems driven by correlated noises: steady-state analysis," Physica A, vol. 388, no. 15-16, pp. 3017-3023, 2009.

[39] D. Guégan, "Chaos in economics and finance," Annual Reviews in Control, vol. 33, no. 1, pp. 89-93, 2009.

[40] K. Aase, B. Øksendal, N. Privault, and J. Ubøe, "White noise generalizations of the Clark-Haussmann-Ocone theorem with application to mathematical finance," Finance and Stochastics, vol. 4, no. 4, pp. 465-496, 2000.

[41] V. V. Konotop and L. Vázquez, Nonlinear Random Waves, World Scientific, River Edge, NJ, USA, 1994.

[42] N. Goldenfeld, Lecture on Phase Transitions and the Renormalization Group, Addison-Wesley, New York, NY, USA, 1992. 


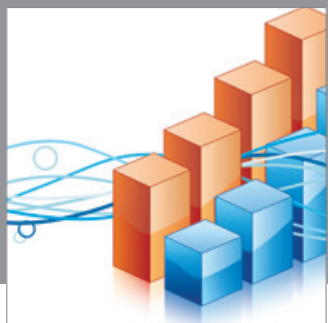

Advances in

Operations Research

mansans

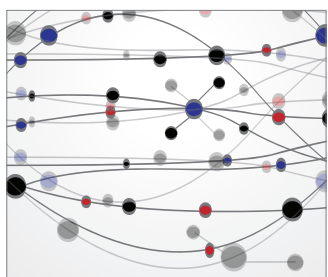

The Scientific World Journal
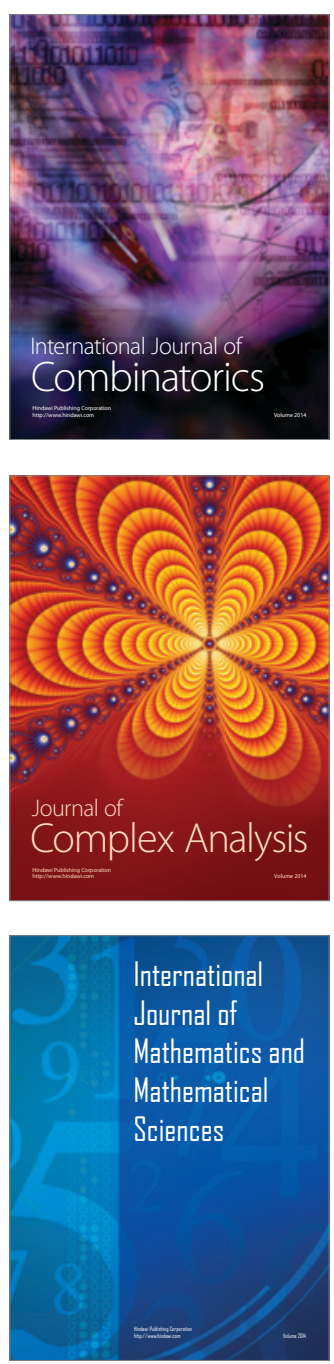
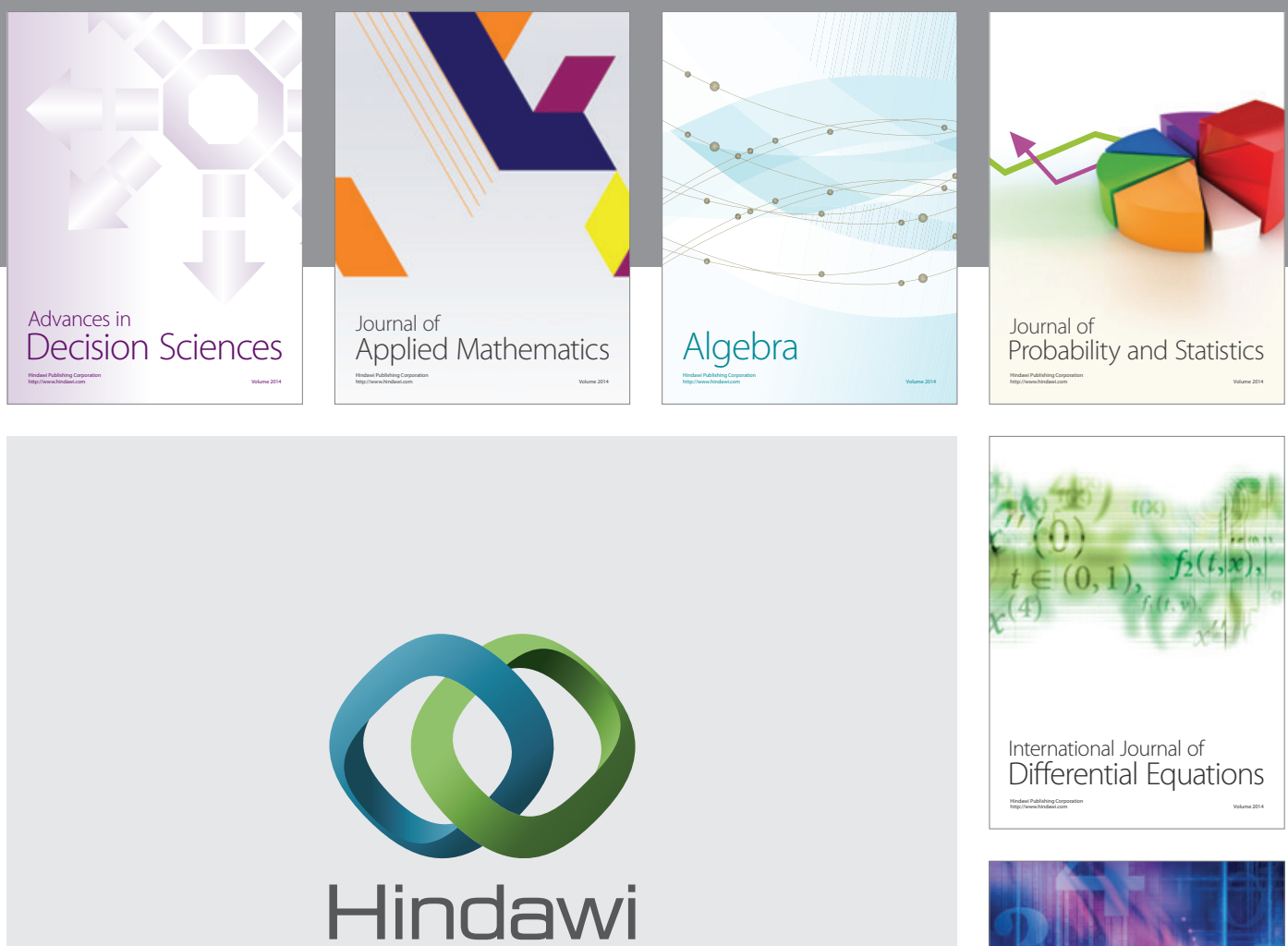

Submit your manuscripts at http://www.hindawi.com
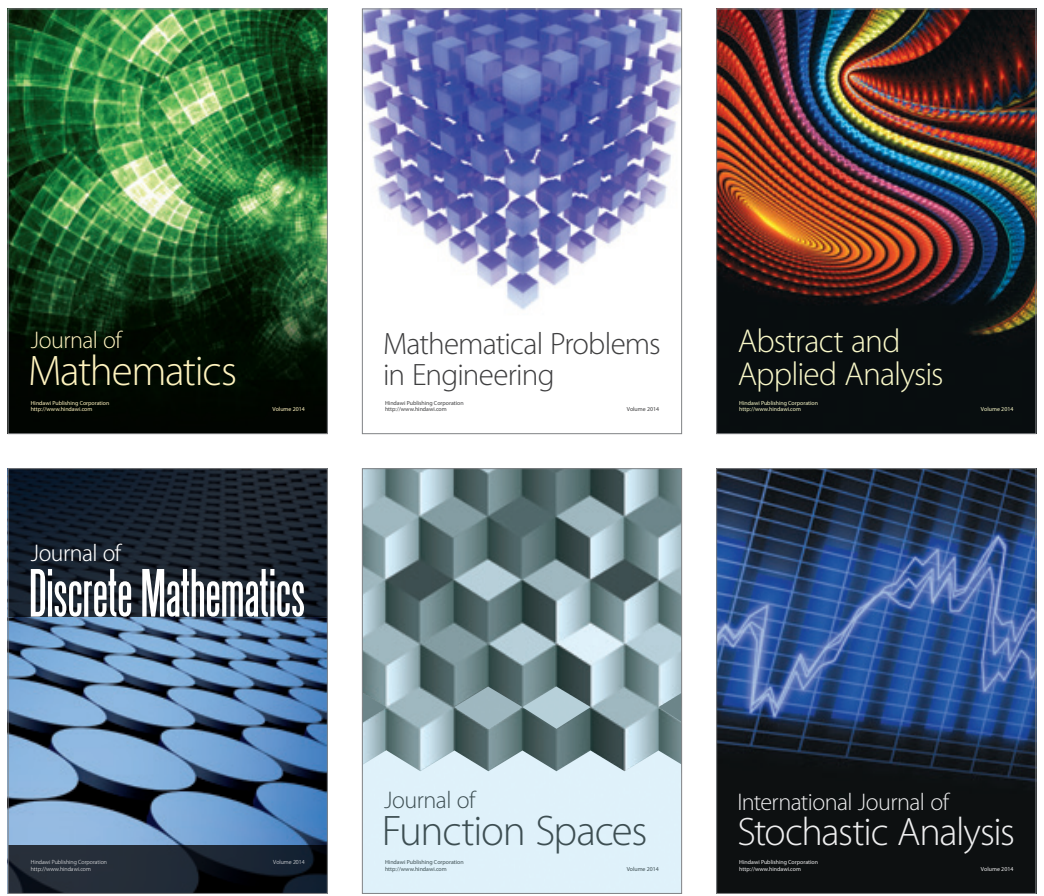

Journal of

Function Spaces

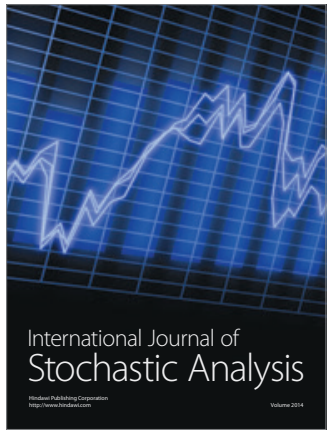

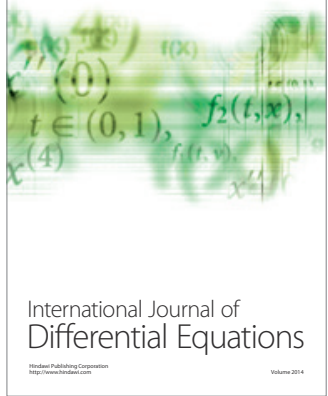
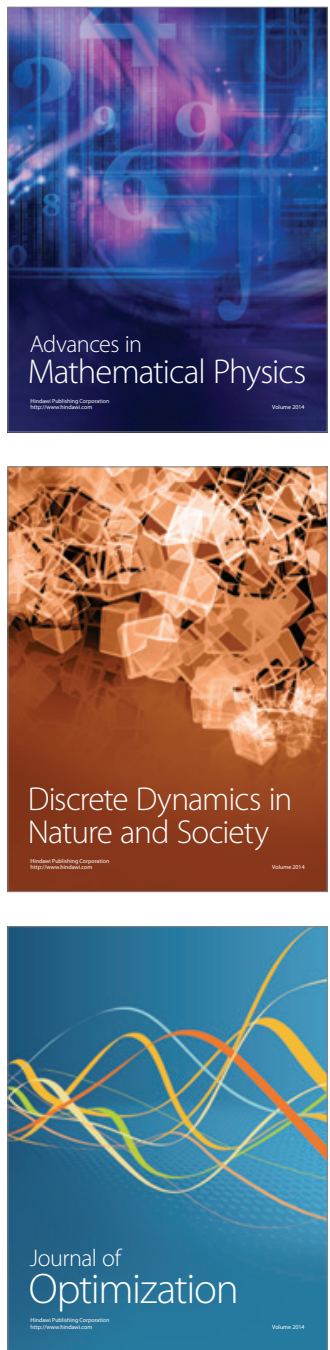